\title{
Sum capacity maximization in distributed multicell MISO-OFDMA systems with reduced feedback links
}

\author{
Berna Özbek ${ }^{1}$, Didier Le Ruyet ${ }^{2}$, Mylene Pischella ${ }^{2}$ \\ ${ }^{1}$ Izmir Institute of Technology, Electrical and Electronics Eng.Dep., Izmir, Turkey. \\ ${ }^{2}$ CEDRIC/LAETITIA, CNAM, Paris, France. \\ e-mail:\{bernaozbek@iyte.edu.tr\} \{didier.le_ruyet, mylene.pischella@cnam.fr\}
}

\begin{abstract}
In this paper, we examine distributed and fair resource allocation for weighted sum multicell capacity maximization in multi-input single-output (MISO)-Orthogonal FrequencyDivision Multiple Access (OFDMA) multicell systems with reduced feedback links. We apply zero forcing (ZF) precoding to reduce interference between the users in the same cell and then propose iterative distributed power allocation algorithm for the users with high signal-to-interference-plus-noise ratio (SINR) to reduce the interference between BSs. In order to perform distributed RA for MISO-OFDMA multicell systems, the channel state information (CSI) of all required links are fed back to all base stations. However, the feedback load increases with the number of users, base stations, subcarriers and antennas. Therefore, we propose a user selection algorithm at the receiver side to reduce the feedback load while providing CSI of the users with high SINR at the BSs. The performance results of the proposed algorithms are illustrated for multicell MISO-OFDMA systems in wireless channels.
\end{abstract}

\section{INTRODUCTION}

The increasing demand for wireless applications requires the design of novel communication systems for high speed, reliable and cost effective transmission solutions. The universal frequency reuse increases peak data rates while leading to high degree of intercell interference (ICI) which arises due to simultaneous transmissions on the same frequency by neighboring base stations (BSs). ICI can significantly reduce data rates in cellular systems especially at cell-edges.

In order to manage ICI, optimal resource allocation (RA) algorithms for multicell orthogonal frequency division multiple access (OFDMA) systems are considered by solving the problem of power and subcarrier allocation jointly in all considered cells. Unfortunately, in most of the practical cases, this global optimization problem is not convex and does not have a simple closed-form solution for multicell OFDMA, in contrast to the single cell OFDMA case. An approach to tackle a variant of this problem with peak power constraint per subcarrier has been presented in [1]. This approach consists to perform a decentralized algorithm that maximizes an upperbound on the sum multicell rate.

Since no exact solution has yet been found, only suboptimal approaches with respect to the optimization criterion exist to tackle it. In [2], a novel distributed algorithm of binary

978-1-4799-5863-4/14/\$31.00 (c) 2014 IEEE power allocation and scheduling for capacity maximization has been presented. In [3], A rate-adaptive joint RA algorithm has been presented by optimizing subcarrier and power allocation iteratively, such that the weighted sum-rate keeps increasing until convergence. Specifically, the algorithm adopts dualitybased methods to optimize the power allocation. In [4], a semidistributed dynamic RA has been presented to suppress ICI, which requires a central unit to perform power allocation.

In the high SINR regime where $\log _{2}(1+$ SINR $) \approx$ $\log _{2}$ (SINR), the weighted sum rate has been solved for single user orthogonal frequency-division multiplexing (OFDM) systems through a decomposition in dual space [5]. Joint allocation of subcarriers and power has been studied in [6] for discrete multitone systems. Their conclusions directly have been extended to downlink multicell OFDMA in [7].

A distributed weighted sum throughput maximization has been performed in [8] via two phases: In the first phase, the users and subcarriers are identified with assumption of uniform power allocation. In the second phase, an iterative distributed algorithm called Dual Asynchronous Distributed Pricing (DADP) [5] has been applied for the remaining users under high SINR assumption. In [9], instead of performing subcarrier allocation by assuming uniform power allocation, a graph based subcarrier allocation has been performed with the combination of distributed power allocation where the weight of each user is determined proportionally to its queue length. This method assumes a semi-distributed multicell network: even though the power allocation is performed distributively by sharing only the price information belonging to each user, the graph based subcarrier allocation requires a centralized unit to build the interference graph.

In order to perform adaptive RA algorithms in multicell systems, the channel state information (CSI) of each user, subcarrier and antenna in each cell is required at the centralized units and/or BSs. A feedback load reduction technique has been examined in [10] by defining different threshold levels for multicell network. Adaptive reduced feedback link design by choosing the users based on their approximate signal to interference noise ratio (SINR) and their locations in the cell has been presented in [11] to satisfy users' rate constraints. In this paper, we propose a distributed RA algorithm for 
multi-input single-output (MISO)-OFDMA multicell systems with reduced feedback link. We perform zero forcing (ZF) precoding seperately at each cell to schedule more than one user for each subcarrier at each cell and propose a distributed iterative power allocation algorithm to reduce the interference between the cells. We rely on high SINR approximation while applying distributed power allocation. Thus, we propose to apply reduced feedback link based on multicell clustered Sbest criterion which guarantees to select clusters with high SINR for each user at each cell.

This paper is organized as follows. In Section II, the system model is described including the optimization problem based on weighted sum multicell capacity maximization. In Section III, we present reduced limited feedback links for multicell MISO-OFDMA systems. In Section IV, we propose the power minimization algorithm for weighted sum multicell capacity maximization. In Section V and VI, the performance results are illustrated and the concluding remarks are drawn, respectively.

\section{System Model}

We consider a downlink MISO-OFDMA multicell system and want to maximize the weighted sum multicell capacity. There are $U$ BSs with $N_{t}$ transmit antennas. At each cell, there are $K$ users with one receive antenna.

First of all, adjacent subcarriers are grouped in a cluster and only one representative value for each cluster is determined for both serving and interfering BSs. The channel coefficient of a cluster is represented by,

$$
\mathbf{h}_{q}=\overline{\mathbf{h}}_{m}
$$

where $\overline{\mathbf{h}}_{m}$ is $N_{t} \times 1$ channel vector of $m$ th subcarrier.

The selection of the representative value is based on the minimum channel gain in the cluster to avoid the outage. Therefore, the value of $m$ is determined as:

$$
m=(q-1) N_{Q}+\arg \min _{1 \leq i \leq N_{Q}}\left\{\left\|\overline{\mathbf{h}}_{(q-1) N_{q}+i}\right\|^{2}\right\} ; \quad \forall q
$$

where $Q$ is the number of clusters, $N_{Q}=M / Q$ is the number of subcarriers in one cluster and $M$ is the number of subcarriers.

The cluster-allocation related notations are defined in the following. The binary variable $a_{u, q}^{k, b} ; b \in\left[1,2, \ldots, N_{t}\right]$ indicates that cluster $q$ is allocated to user $k$ for antenna $b$ at BS $u$ if $a_{u, q}^{k, b}=1 .\left\{a_{u, q}^{k, b}\right\}_{q=1}^{Q}$ for each antenna is stacked into the vector $\mathbf{a}_{u}^{k}=\left[a_{u, 1}^{k, 1}, \ldots, a_{u, 1}^{k, N}, \ldots, a_{u, Q}^{k, 1}, \ldots, a_{u, Q}^{k, N}\right]^{T}$ and then $\left\{\mathbf{a}_{u}^{k}\right\}_{k=1}^{K}$ is stacked to a matrix $\mathbf{A}_{u}$ column by column. Finally, a matrix $\mathbf{A}=\left[\mathbf{A}_{1}, \ldots, \mathbf{A}_{U}\right]$ indicates how the users are allocated to the clusters for all antennas and all cells.

The power-allocation related notations are defined in the following. $p_{u}^{\max }$ denotes the total available power at $\mathrm{BS} u$, $p_{u, q}$ represents the allocated power and $p_{u, q}^{\max }$ is the maximum allowed power level to cluster $q$ by BS $u$. $\left\{p_{u, q}\right\}_{u=1}^{U}$ is stacked into a $U \times 1$ vector $\mathbf{p}_{q}=\left[p_{1, q}, p_{2, q}, \ldots, p_{U, q}\right]^{T}$ and then the power allocation matrix $\mathbf{P}$ with $U \times Q$ is constructed.
Considering that the optimization variables are the set of clusters $\mathbf{A}$ and the set of power values $\mathbf{P}$, the weighted sum multicell capacity maximization RA problem is defined as:

$$
\max _{\mathbf{A}, \mathbf{P}} \sum_{u=1}^{U} \sum_{k=1}^{K} \sum_{q=1}^{Q} \sum_{b=1}^{N} a_{u, q}^{k, b}\left(m_{u}^{k} R_{u, q}^{k, b}\left(p_{u, q}\right)\right)
$$

subject to

$$
\begin{aligned}
\sum_{q=1}^{Q} p_{u, q} & =p_{u}^{\max } \quad \forall u \\
0 \leq p_{u, q} & \leq p_{u, q}^{\max } \quad \forall u, \forall q \\
\sum_{k=1}^{K} a_{u, q}^{k, b} & \leq 1 \quad \forall u, \forall q, \forall k, \forall b \\
a_{u, q}^{k, b} & \in\{0,1\} \quad \forall u, \forall q, \forall k, \forall b
\end{aligned}
$$

where $m_{u}^{k}$ is weight of $k$ th user at $u$ th cell, $\left(C_{1}\right)$ and $\left(C_{2}\right)$ are constraints for power allocation, $\left(C_{3}\right)$ and $\left(C_{4}\right)$ are constraints for cluster allocation. The power is equally assigned to each antenna such that $p_{u, q}^{b}=p_{u, q} / N_{t}$.

In order to provide fairness between users in the cell, the weight of each user is determined proportional to its location in the cell. Therefore, when the user is far from the BS, its weight becomes large and it increases its scheduling chance in the RA algorithm. Then, we set $m_{u}^{k}$ as the normalized distance, $d_{u}^{k} / R$ where $d_{u}^{k}$ is the distance between user $k$ and BS $u$ and $R$ is the radius of the cell.

The data rate of the $k$ th user at BS $u$ in the $q$ th cluster is calculated as,

$$
R_{u, q}^{k, b}\left(p_{u, q}\right)=\frac{B}{Q} \log _{2}\left(1+\gamma_{u, q}^{k, b}\left(p_{u, q}\right)\right)
$$

where $B$ is the total available bandwidth.

The SINR, $\gamma_{u, q}^{k, b}\left(p_{u, q}\right)$, is determined by,

$$
\gamma_{u, q}^{k, b}\left(p_{u, q}\right)=\frac{\frac{p_{u, q}}{N}\left|\left(\mathbf{h}_{u, u, q}^{k}\right)^{H} \mathbf{w}_{u, q}^{b}\right|^{2}}{\frac{N_{0} B}{Q}+U_{u, q}^{k, b}+C_{u, q}^{k}}
$$

where $N_{0}$ is the power noise density and $\mathbf{h}_{u, u, q}^{k}=$ $\left[h_{u, u, q}^{k, 1}, \ldots, h_{u, u, q}^{k, N}\right]^{T}$ is the channel vector of user $k$ with the channel coefficient $h_{u, u, q}^{k, b}$ that belongs to user $k$ at BS $u$ for cluster $q$ from antenna $b$ in cell $u$ and includes path loss and multipath effect of the wireless channels. Note that if $u \neq v, h_{u, v, q}^{k, b}$ represents the channel coefficient of the cochannel interfering link from antenna $b$ of BS $v$ to the user $k$ located in cell $u$. It is assumed that the wireless channel between each BS-user pair remains static over a sufficiently long duration to perform RA.

In order to avoid inter-user interference (IUI) at each cell, the precoding matrix $\mathbf{W}_{u, q}$ is obtained by using $\mathrm{ZF}$ by,

$$
\mathbf{W}_{u, q}=\beta_{u, q}\left(\mathbf{H}_{u, q}\right)^{H}\left[\left(\mathbf{H}_{u, q}\right)\left(\mathbf{H}_{u, q}\right)^{H}\right]^{-1}
$$

where $\mathbf{H}_{u, q}=\left[\mathbf{h}_{u, u, q}^{1}, \ldots, \mathbf{h}_{u, u, q}^{K}\right]^{T}$ denotes the matrix of $K \times N_{t}$ consisting of the stacked channel vectors of the pair 
of assigned users for each cluster at each cell and $\beta_{u, q}$ is calculated by,

$$
\beta_{u, q}=\frac{1}{\sqrt{\operatorname{tr}\left[\left(\left(\mathbf{H}_{u, q}\right)\left(\mathbf{H}_{u, q}\right)^{H}\right)^{-1}\right]}}
$$

$\mathbf{w}_{u, q}^{b}$ is the $b^{\text {th }}$ column of precoding matrix $\mathbf{W}_{u, q}$.

IUI is calculated by,

$$
U_{u, q}^{k, b}=\frac{p_{u, q}}{N_{t}} \sum_{j=1 ; j \neq b}^{N}\left|\left(\mathbf{h}_{u, u, q}^{k}\right)^{H} \mathbf{w}_{u, q}^{j}\right|^{2}
$$

ICI is determined by,

$$
C_{u, q}^{k}=\sum_{v=1, v \neq u}^{U} \frac{p_{v, q}}{N_{t}} \sum_{j=1}^{N}\left|\left(\mathbf{h}_{u, v, q}^{k}\right)^{H} \mathbf{w}_{v, q}^{j}\right|^{2}
$$

In the following, to simplify notations, we denote

$$
G_{u, v, q}^{k, b}=\left|\left(\mathbf{h}_{u, v, q}^{k}\right)^{H} \mathbf{w}_{v, q}^{b}\right|^{2}
$$

and

$$
\widetilde{G}_{u, v, q}^{k}=\sum_{j=1}^{N} G_{u, v, q}^{k, j}
$$

Consequently, ICI is equivalently written as:

$$
C_{u, q}^{k}=\sum_{v=1, v \neq u}^{U} \frac{p_{v, q}}{N_{t}} \widetilde{G}_{u, v, q}^{k}
$$

Then, the SINR expression becomes:

$$
\gamma_{u, q}^{k, b}\left(p_{u, q}\right)=\frac{\frac{p_{u, q}}{N} G_{u, u, q}^{k, b}}{\frac{N_{0} B}{Q}+U_{u, q}^{k, b}+\sum_{v=1, v \neq u}^{U} \frac{p_{v, q}}{N} \widetilde{G}_{u, v, q}^{k}}
$$

\section{THE REDUCED FEEDBACK LINKS}

We propose to perform a cluster selection for each user at each cell based on approximate SINR criterion [11][12]. The clusters are selected by assuming that full power is assigned to each BS and is shared equally between the clusters. The precoding vector is designed under the condition that there is no cooperation between the users and consequently only one user is assigned and IUI does not occur.

The approximate SINR value of each user at each cluster for multicell system is calculated as:

$$
\tilde{\gamma}_{u, q}^{k}=\frac{\frac{p_{u}^{\max }}{Q}\left\|\mathbf{h}_{u, u, q}^{k}\right\|^{2}}{\frac{N_{0} B}{Q}+\tilde{C}_{u, q}^{k}}
$$
where $\tilde{C}_{u, q}^{k}=\sum_{v=1, v \neq u}^{U} \frac{p_{u}^{\max }}{Q}\left\|\mathbf{h}_{u, v, q}^{k}\right\|^{2}$ is the interference
power.

At each cell, each user constructs independently a set $\mathbb{S}_{u}^{k}$ composed of the strongest $S_{u}^{k}$ clusters based on $\tilde{\gamma}_{u, q}^{k}$ values.

Let $\mathbb{T}_{u, q}$ be the set of users that feeds back the CSI associated to the cluster $q$ at cell $u$ as,

$$
\mathbb{T}_{u, q}=\left\{k \in\{1,2, \ldots, K\}: q \in \mathbb{S}_{u}^{k}\right\}
$$

Then, each user feeds back the CSI belonging to these selected clusters for both serving and interfering BSs to its own BS.

The determination of the number of clusters, $S_{u}^{k}$, is very critical for system performance.

In order to provide fairness between users, we propose to set a specific weight for each user according to their location in the cell and determine the same number of clusters for each user by setting $S_{u}^{k}=S$. The $S$ value is defined as a function of the feedback load and/or the number of users in the multicell systems. Consequently, total feedback load is proportional to $K S$ at each cell.

\section{Distributed Resource Allocation}

In this section, we perform user scheduling based on $\mathrm{ZF}$ precoding and propose a distributed RA algorithm for multicell network, which aims at maximizing the weighted sum multicell capacity.

Assuming that the channel vectors $\mathbf{h}_{u, u, q}^{k} ; \forall u$ for $k \in \mathbb{T}_{u, q}$ is available at BS $u$ perfectly, we select up to $N_{t}$ users to maximize weighted sum multicell capacity at each BS separately.

For each cluster at each cell, we select the users by,

$$
\mathbf{A}_{u, q}^{*}=\arg \max \sum_{b=1}^{N} m_{u}^{k} \log _{2}\left(1+\gamma_{u, q}^{k, b}\right) \quad k \in \mathbb{T}_{u, q}
$$

where

$$
\gamma_{u, q}^{k, b}=\frac{p_{u}^{\max }}{N_{0} B}\left|\left(\mathbf{h}_{u, u, q}^{k}\right)^{H} \mathbf{w}_{u, q}^{b}\right|^{2}
$$

and the precoding vectors are determined according to Eq. (6).

We assume that the CSI belonging to $\mathbf{h}_{u, v, q}^{k} ; \forall u, v$ for $k \in \mathbf{A}_{u, q}^{*}$ are perfectly available at all BSs. Then, we perform the proposed distributed power allocation algorithm based on DADP method [5], adapted to the MISO case and to the proposed clustering.

Since $\mathrm{ZF}$ is performed at each cell separately, the IUI is removed completely and then we get:

$$
\gamma_{u, q}^{k, b}\left(p_{u, q}\right)=\frac{\frac{p_{u, q}}{N} G_{u, u, q}^{k, b}}{\frac{N_{0} B}{Q}+\sum_{v=1, v \neq u}^{U} \frac{p_{v, q}}{N} \widetilde{G}_{u, v, q}^{k}}
$$

Let $k \in \Omega_{q}$ be the set of interfering users in cluster $q$. We suppose that the high SINR assumption $\log _{2}(1+$ SINR $) \approx$ $\log _{2}$ (SINR) is valid thanks to the pre-selection of the users at the receiver side according to proposed reduced feedback links, the power optimization problem is given by,

$$
\max _{\mathbf{P}} \sum_{q=1}^{Q} \sum_{b=1}^{N} \sum_{k \in \Omega_{q}} m_{u}^{k} \log _{2}\left(\gamma_{u, q}^{k, b}\left(p_{u, q}\right)\right)
$$

subject to constraints given in $C_{1}$ and $C_{2}$.

This is equivalent to:

$$
\max _{\mathbf{P}} \prod_{q=1}^{Q} \prod_{b=1}^{N} \prod_{k \in \Omega_{q}}\left(\gamma_{u, q}^{k, b}\left(p_{u, q}\right)\right)^{m_{u}^{k}}
$$


subject to constraints given in $C_{1}$ and $C_{2}$.

Then, we perform the following distributed power allocation algorithm:

Initialization: At $T_{d}=0$, set the initial power and price for all clusters $q$ and all users $k \in \Omega_{q}$, and the initial dual price per BS $\delta_{u}(0) \geq 0, \forall u \in\{1, \ldots, U\}$.

\section{Iterative process:}

1) Dual price update: At each iteration $T_{d}$, each BS $u \in$ $\{1, \ldots, U\}$ updates its dual price according to:

$$
\delta_{u}\left(T_{d}\right)=\max \left\{\delta_{u}\left(T_{d}-1\right)+Y, 0\right\}
$$

where $Y=\kappa\left(\sum_{q=1}^{Q} p_{u, q}\left(T_{d}-1\right)-p_{u}^{\max }\right)$.

2) Iterative power and interference information update:

For a given dual price setting, an iterative process is used independently on each cluster $q \in\{1, \ldots, Q\}$.

At iteration $T=0, \forall k \in \Omega_{q}$, set $p_{u, q}(0)=p_{u, q}^{\max }$, and compute the corresponding interference information:

$$
\zeta_{u, q}^{k}(0)=\frac{m_{u}^{k}}{I_{u, q}^{k}(0)}
$$

where the interference plus noise term received by the user $k$ served by $\mathrm{BS} u$ in cluster $q$ at time 0 is

$$
I_{u, q}^{k}(0)=\frac{N_{0} B}{Q}+\sum_{v \neq u} \frac{p_{v, q}(0)}{N_{t}} \widetilde{G}_{u, v, q}^{k}
$$

Inner iterative process:

a) Power update:

Compute the power of each user $k \in \Omega_{q}$, depending on its channel state, weight, the interference information of the previous iteration, and on the dual price of its serving BS $u, \delta_{u}^{k}\left(T_{d}\right)$ :

$$
\begin{aligned}
& p_{u, q}(T+1)=0, \text { if } Y_{u, q}^{k, b}\left(\mathbf{P}_{q}(T)\right) \leq 0 \\
& p_{u, q}(T+1)=p_{u, q}^{\max }, \text { if } Y_{u, q}^{k, b}\left(\mathbf{P}_{q}(T)\right) \geq p_{u, q}^{\max } \\
& p_{u, q}(T+1)=Y_{u, q}^{k, b}\left(\mathbf{P}_{q}(T)\right), \text { otherwise }
\end{aligned}
$$

where

$$
Y_{u, q}^{k, b}\left(\mathbf{P}_{q}(T)\right)=\frac{m_{u}^{k}}{\left(\sum_{v \neq u} \widetilde{G}_{v, u, q}^{k} \zeta_{v, q}^{k}(T)+\delta_{u}^{k}\left(T_{d}\right)\right)}
$$

b) Interference information update: Compute the interference information of each user $k \in \Omega_{q}$ served by $\mathrm{BS} u$, depending on its weight and received noise plus interference,

$$
\zeta_{u, q}^{k}(T+1)=\frac{m_{u}^{k}}{I_{u, q}^{k}(T+1)}
$$

where $I_{u, q}^{k}(T+1)=\frac{N_{0} B}{Q}+\sum_{v \neq u} \frac{p_{v, q}(T+1)}{N} \widetilde{G}_{u, v, q}^{k}$.

This algorithm converges to the global optimum of problem (20). The convergence proof is similar to the one used for multi-channel DADP in [5]. It is based on the fact that the studied optimization problem belongs to the class of geometric programming [13] and can consequently be solved by Lagrangian relaxation. The dual price update can be viewed as a distributed gradient projection algorithm for solving the master problem. This algorithm converges for small enough step size $\kappa$. The proof of [5] applies to our case even if, contrary to case of [5], we consider several users per cell, as the users served by the same BS are only differentiated by their channel gains, and the sum power constraint applies to the whole cell.

\section{Performance Results}

We obtain the performance results to illustrate the benefits of the multicell MISO-OFDMA system with $N_{t}=2$ transmit antennas for $U=3$ BSs through wireless channels. The users are uniformly distributed in a multicell system where the radius of each cell is $750 \mathrm{~m}$. The maximum transmitted power, $p_{u}^{\max } ; \forall u$ and the noise power density are set at $43.10 \mathrm{dBm}$ and $-174 \mathrm{dBm} / \mathrm{Hz}$ respectively. The path loss model is $L_{p}=128.1+37.6 \log _{10}(d(\mathrm{~km})) \mathrm{dB}$ and the wireless channel is modeled using 3GPP-TU. The bandwidth and the carrier frequency are selected as $10 \mathrm{MHz}$ and $2.4 \mathrm{GHz}$. The number of clusters is set to $Q=40$ while grouping 12 subcarriers in each cluster.

The feedback load defines as the total number of feedback clusters is shown in Fig. 1 for different $S$ values. Depending on the chosen $S$, the feedback load is reduced of $40 \%$ to $60 \%$ compared to the full feedback case. For the corresponding $S$ values, the simulation results in terms of sum and weighted sum multicell capacity are illustrated in Fig.2. As shown in the performance evaluations, the sum multicell capacity results is approximately $3 \mathrm{bps} / \mathrm{Hz}$ lower than full feedback, which represents a decrease of $12.5 \%$, whereas the feedback load is reduced of $40 \%$. However, the weighted sum multicell capacity results with reduced feedback link are approximately the same as in the full feedback case while reducing the feedback load significantly.

In Fig.3, the fairness performance of the algorithms is given according to Jain index where The FI ranges between 0 (no fairness) and 1 (perfect fairness):

$$
\mathrm{FI}=\frac{\left(\sum_{k=1}^{K} R_{u}^{k}\right)^{2}}{K \sum_{k=1}^{K}\left(R_{u}^{k}\right)^{2}}
$$

The weighted sum multicell capacity algorithm provides a fairness index around 0.9 in which all users achieve almost the same data rate regardless of their location in the cell while the fairness performance of sum multicell capacity algorithm is very poor. In addition to that the proposed reduced feedback links slightly improves the fairness performance while giving almost the same sum capacity performance. 


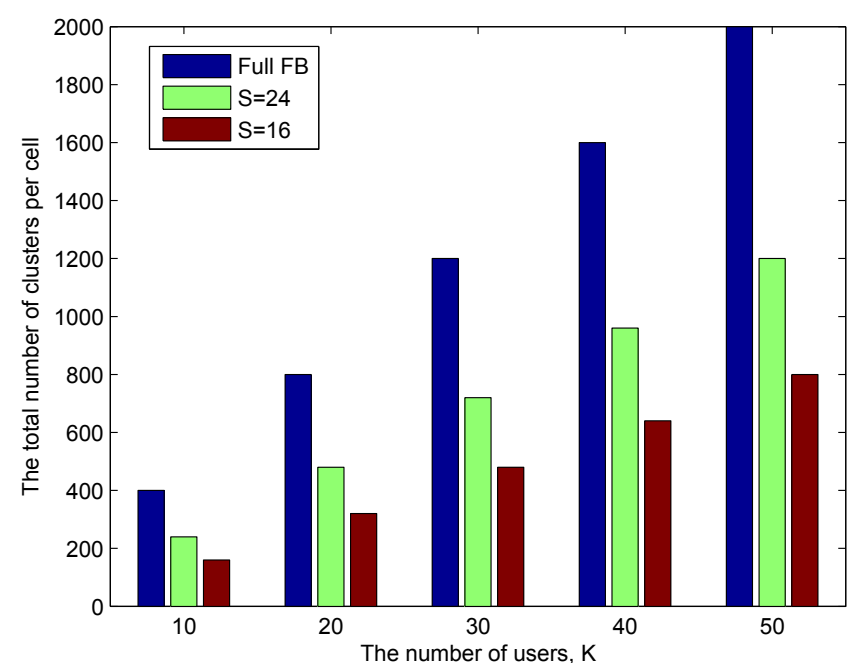

Fig. 1. Feedback load of MISO-OFDMA multicell systems.

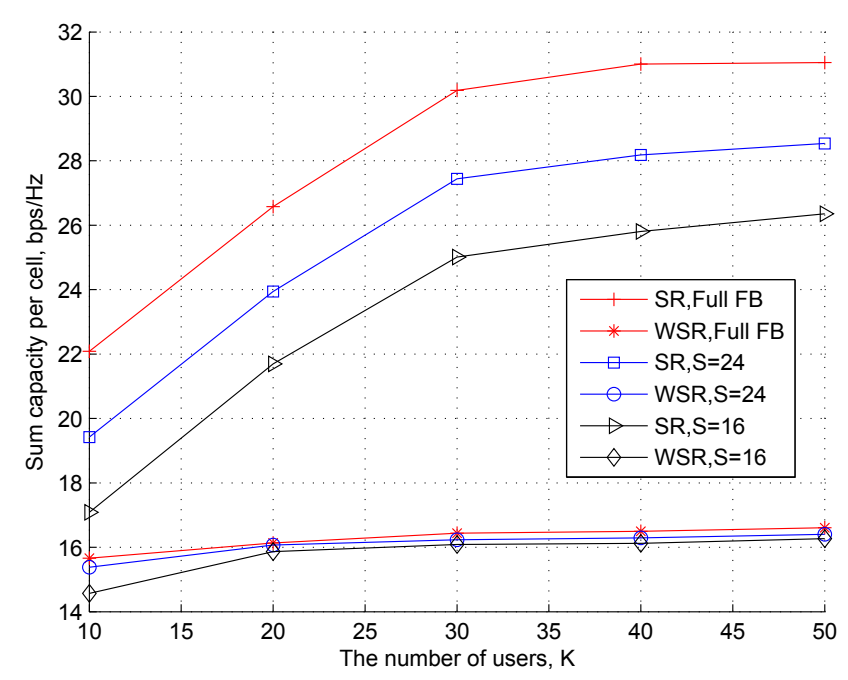

Fig. 2. Sum capacity performance of MISO-OFDMA multicell systems.

\section{CONCLUSION}

In this paper, we have proposed distributed multicell MISOOFDMA systems with reduced feedback links. The iterative power allocation algorithm has been derived for weighted sum capacity maximization in multiuser multicell multicarrier scenario. In order to reduce the feedback load, we perform a user selection algorithm at the user side based on multicell clustered S-best criterion. While performing distributed power allocation algorithm we rely on the high SINR approximation since the proposed reduced feedback links are guaranteed to provide the selection of users with high SINR. We have reduced the feedback load significantly while providing fairness and almost the same sum capacity performance for distributed multicell MISO-OFDMA systems.

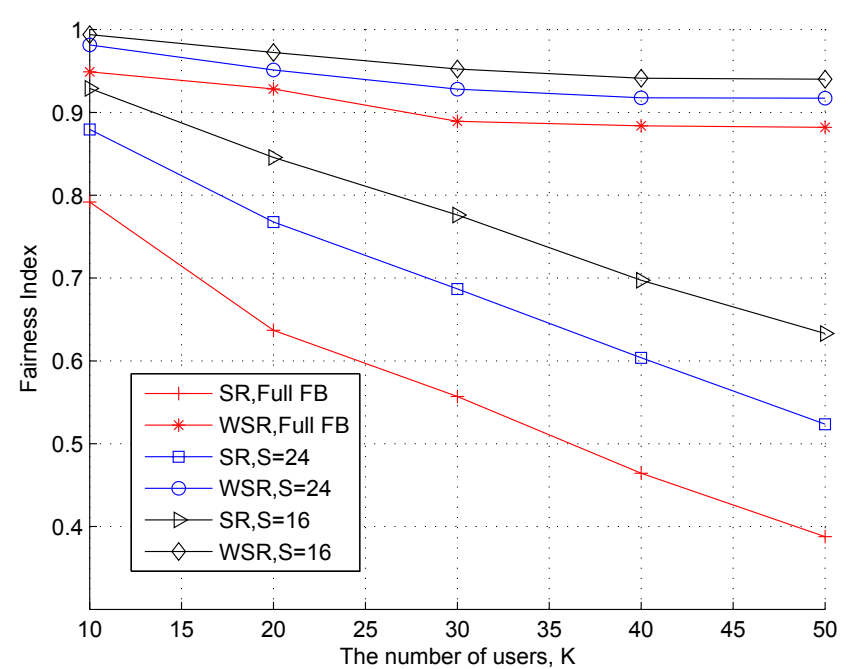

Fig. 3. Fairness performance of MISO-OFDMA multicell systems.

\section{REFERENCES}

[1] D. Gesbert and M. Kountouris, "Rate scaling laws in multicell networks under distributed power control and user scheduling", IEEE Transactions on Information Theory, Jan. 2011, pp.234-244.

[2] S. Kiani and G. Oien and D. Gesbert, "Maximizing multicell capacity using distributed power allocation and scheduling", in Proc. of Wireless Communications Networking Conference, pp. 1690-1694, March 2007.

[3] L. Venturino and N. Prasad and X. Wang, "A successive convex approximation algorithm for weighted sum-rate maximization in downlink OFDMA networks", in Proc. of Conference on Information Sciences Systems, pp. 379-384, July 2008.

[4] Z. Chongxian and L. Chunguo and Y. Luxi and H. Zhenya, "Dynamic resource allocation for the downlink of multi-cell systems with full spectral reuse", in International Conference on Neural Networks and Signal Processing, pp. 173-177, July 2008.

[5] J. Huang, R. Berry and M.L. Honig, "Distributed interference compensation for wireless networks", in IEEE J. Select. Areas Commun., vol. 24, no. 5, pp. 1074-1084, May 2006.

[6] W. Yu and R. Lui, "Dual methods for nonconvex spectrum optimization of multicarrier systems", in IEEE Transactions on Communications, pp. 1310-1322, July 2006.

[7] L. Venturino and N. Prasad and X. Wang, "Coordinated scheduling and power allocation in downlink multicell OFDMA networks", in IEEE Transactions on Vehicular Technology, vol. 58, no. 6, pp. 2825-2834, July 2006.

[8] M. Pischella and J.-C. Belfiore, "Distributed weighted sum throughput maximization in multi-cell wireless networks", in IEEE 19th International Symposium on Personal, Indoor and Mobile Radio Communications (PIMRC), September 2008.

[9] M. Pischella and J.-C. Belfiore, "Weighted sum throughput maximization in multi-cell OFDMA networks", IEEE Transactions on Vehicular Technology, vol:59, no:2, pp:896-905, February 2010.

[10] A. Papadogiannis, H. J. Bang, D. Gesbert, E. Hardouin, "Efficient Selective Feedback Design for Multicell Cooperative Networks", IEEE Transaction on Vehicular Techology., vol:60, no:1, pp:196 - 205, Jan. 2011.

[11] B. Ozbek, D. Le Ruyet, M. Pischella, "Adaptive reduced feedback links for distributed power allocation in multicell MISO-OFDMA networks", IEEE Wireless Communications Letters, vol:3 , Issue: 2, pp. 141 - 144, April 2014.

[12] B. Ozbek, D. Le Ruyet, "Feedback strategies for wireless communication", Springer-Engineering Series Book, Springer, New York, U.S.A, 2013.

[13] M. Chiang, Geometric programming for communication systems, Now Publishers (Fundations and trends in communications and information theory), 2005. 